\title{
PAYS-BAS
}

DES hOYENS DE RENDRE VISIBLE A DISTANGE LE SIGNE DE LA CROIX-ROUGE EN TEMPS DE GUERRE

Préoccupé du danger que courent à la guerre les ambulances et les transports de la Croix-Rouge à cause de l'insuffisance habituelle du signe protecteur de la Croix-Rouge, M. le Dr C. de Mooy, médecin principal de $1^{\text {re }}$ classe en retraite, membre du Comité supérieur de la Croix-Rouge néerlandaise, un vétéran bien connu des amis de la Croix-Rouge, s'est ingénié à trouver un moyen pour que, de loin, l'ennemi soit toujours et facilement renseigné sur la présence d'une ambulance ou d'un convoi de la Círoix-Rouge lorsqu'ils arriveraient à se trouver dans sa ligne de feu.

On doit constater, en effet, que le tir à longue portée de nos armes modernes place les secours pendant le combat dans une situation bien précaire, quand précisément, la nature même des blessures modernes rendrait des plus désirables une très prompte intervention. La proposition de M. le $\mathrm{D}^{\mathrm{r}}$ de Mooy, "de modifier et de remplacer les drapeaux ambulanciers par des plaques ou disques visibles », mérite donc toute l'attention des services sanitaires '.

L'auteur commence par constaler très logiquement que le signe de la Croix-Rouge, sur les voitures d'ambulance, se trouve généralement placé sur les deux longs côtés de la voiture seulement et qu'ainsi on ne le remarque ni de devant ni de derrière. Quant aux drapeaux, s'il n'y a pas de vent ou s'ils sont mouillés, ils restent collés le long de la hampe et ne sont de ce fait guère reconnaissables. Il en est de même lorsque le vent souffle dans le sens de ceux qui regardent et présente ainsi les drapeaux sagittalement à l'œil de l'observateur. Ces circonstances ont été plus d'une fois la cause d'erreurs malheureuses, qui, non seulement, ont mis en danger les malades et le personnel des ambulances et des trains sanitaires, mais ont en outre provoqué des accusations injustes, en présentant l'ennemi comme ayant attaqué volontairement des convois on des

1 Résumé d'un rapport présenté à la réunion du Comité supérieur de la. Croix-Rouge, à La Haye, le 27 janvier 1903, et envoyé en manuscrit an Comité international par l'auteur.

Comparez l'article que nous avons publié en 1900, T. XXXI, p. 180. 
installations protégés par le signe de la Croix-Rouge, cela en violation flagrante des clauses de la Convention de Genève.

M. de Mooy propose donc de remplacer les drapeaux de la CroixRouge par des plaques fixes, soit en métal soit en toile forte sur cadre, ou par des disques mobiles bien visibles. La nuit on éclairerait les plaques au moyen de projecteurs à acétylène, on bien on employerait de fortes lanternes avec verres rouges en croix sur les quatre faces. Toutes les ambulances, voitures, trains, bateaux et barques servant aux malades et blessés seraient munis obligatoirement de ces plaques de la Croix-Rouge de grandeurs suffisantes et visibles en toutes circonstances grâce à une adaptation convenable. Pour rendre, en outre, reconnaissable de fort loin, au besoin, le signe protecteur de la Croix-Rouge, M. le $\mathrm{D}^{\mathrm{r}}$ de Mooy propose de le fixer, à l'occasion, à des ballons captifs, servant la nuit, du même coup, à l'éclairage. Ce serait l'aérostation mise au service de la Croix-Rouge !

M. de Mooy nous paraît avoir fort utilement attiré l'attention sur un point d'organisation des ambulances militaires ou volontaires qui peut avoir éventuellement une importance très grande pour la sécurité des blessés dans les combats modernes.

Dr FerRìike.

\section{TRAITEMENT DES PLAIES EN CAMPAGNE}

Il n'est pas sans intérêt de relever les expériences des médecins qui onl suivi la campagne récente. Résumons à cet égard, brièvement, la thèse de $\mathrm{M}$. le $\mathrm{D}^{\mathrm{r}}$ Juriaanse, fruit de ses expériences après deux ans et demi passés sur les champs de bataille sud-africains, à côté des Boers '.

L'auteur est d'avis qu'on n'a pas encore créé une organisation des ambulances qui suffise sur tous les points; les guerres antérieures ne peuvent rien nous apprendre à cause de la transformation complète dans l'art de faire la guerre. L'organisation du service de santé chez les Boers laissait tout à désirer, celle des Anglais était assez bonne. L'auteur décrit en détail ce que doit

1 Veldambulances en Wondbehandeling te velde. Nous extrayons l'analyse résumée qui suit d'un article de M. le $\mathrm{D}^{\mathrm{r}}$ Janssen dans le Caducée du 18 arril $1903\left(n^{\circ} 8\right.$, p. 101). 